\title{
$\mathrm{MCMC}$ 방법을 이용한 자율주행 차량의 보행자 탐지 및 추적방법
}

\section{Pedestrian Detection and Tracking Method for Autonomous Navigation Vehicle using Markov chain Monte Carlo Algorithm}

\begin{abstract}
황 중 원 ${ }^{1}$, 김 남 훈 ${ }^{1}$, 윤 정 연 ${ }^{1}$, 김 창 환 ${ }^{\dagger}$
Jungwon Hwang ${ }^{1}$, Namhoon Kim ${ }^{1}$, JeongYeon Yoon ${ }^{1}$, ChangHwan Kim ${ }^{\dagger}$

Abstract In this paper we propose the method that detects moving objects in autonomous navigation vehicle using LRF sensor data. Object detection and tracking methods are widely used in research area like safe-driving, safe-navigation of the autonomous vehicle. The proposed method consists of three steps: data segmentation, mobility classification and object tracking. In order to make the raw LRF sensor data to be useful, Occupancy grid is generated and the raw data is segmented according to its appearance. For classifying whether the object is moving or static, trajectory patterns are analysed. As the last step, Markov chain Monte Carlo (MCMC) method is used for tracking the object. Experimental results indicate that the proposed method can accurately detect moving objects.
\end{abstract}

Keywords: Detection and Tracking of Moving Objects, Markov Chain Monte Carlo(MCMC)

\section{1. 서 론}

최근 로봇이나 차량이 자율적으로 주행하게 하기 위하여 데이터를 수집하고, 처리하고, 이를 이용하여 하드웨어를 제어하는 많은 연구가 진행되고 있다. 그러 한 많은 연구 중에 장애물을 찾는 것과 관련된 연구는 매우 기본적인 연구이며 중요한 연구이다. 장애물을 찾 는 방법은 이용하는 센서에 따라 레이저 거리 센서를 이용하는 방법과 ${ }^{[1-4]}$ 카메라를 이용하는 방법 ${ }^{[5,6]}$ 으로 나 눌 수 있다. 또한 움직이는 객체를 찾는 방법과 ${ }^{[1-6]}$ 움 직임을 구분하지 않고 객체를 찾는 방법으로 ${ }^{[7]}$ 나눌 수 있다. 본 연구는 여러 연구 주제 중, 레이저 거리

Received : Mar. 6. 2012; Reviewed : Mar. 22. 2012; Accepted: Apr. 20. 2012 ※ This work was supported by the R\&D program of the Korea Ministry of Knowledge and Economy (MKE) and the Korea Evaluation Institute of Industrial Technology (KEIT). (The Development of Low-cost Autonomous Navigation Systems for a Robot Vehicle in Urban Environment, 10035354)

+ 교신저자 : 한국과학기술연구원 책임연구원

${ }^{1}$ 한국과학기술연구원
센서만을 이용하여 움직이는 객체를 찾는 방법에 관한 연구이다.

레이저 센서를 이용하여 이동 객체를 찾는 방법 중, 가장 대표적인 방법은 C.C.Wang이 제안한 SLAMMOT (SLAM with Moving Objects Tracking) 방ㅂㅂㅂ ${ }^{[2]}$ 이다. 이 방법은 SLAM 방법에서 정적인 객체와 동적인 객 체를 구분하여, 이동 차량의 위치 정보 파악과 (Localization) 이동 객체 정보를 모두 찾아내는 방법이 다. 이 방법은 실시간으로 정보를 처리할 수 있는 장 점도 가진다. 그러나 정적 정보를 활용하기 위한 충분 한 데이터가 없는 경우 즉, 이동 객체가 매우 많은 경 우에는 적용하기 매우 어렵다는 단점을 가진다. 본 연 구는 위치 정보를 파악하는 방법과 이동 객체를 찾아 내는 방법을 혼합하지 아니하고, 파악된 위치정보를 사 용하여 이동 객체를 찾는 방법을 제안한다. 이를 위하 여 T. Mori가 제안하는 방법 ${ }^{[9]}$ 인 물체의 이동 궤적을 이용하는 방법을 사용하여 이동 물체는 찾는 방법을 제안한다. 
또한 본 논문에서 제안하는 방법의 처리 속도를 향 상시키기 위하여 물체를 추적하는 방법에 Markov chain Monte Carlo(MCMC) 알고리즘을 이용하여 계산 복잡도를 감소시키는 방법을 제안한다.

본 논문의 구성은 다음과 같다. 2장에서는 객체 모 델을 정의하고 이를 탐지하는 방법을 기술하며, 3 장에서 는 객체의 이동성을 측정하는 방법을 제안한다. 4 장에서 는 탐지된 객체를 추적하는 방법을 제안하고, 5 장에서는 실험 결과를 분석하고, 6장에서는 결론을 맺는다.

\section{2. 객체 모델}

먼저, 이 장에서는 객체 모델을 형성하기 위하여 격자지도를 생성하는 방법과 본 연구에서 제안하는 보 행자 모델을 제시한다.

\section{1 격자 지도 생성}

자율주행 차량에서 이동 방향 전방에 있는 객체를 찾기 위하여 레이저 거리 센서(Laser Range Finder: $\mathrm{LRF}$ ) 데이터를 사용한다. 거리 데이터만을 가지고 객 체를 파악할 경우, 계산 복잡도가 매우 높고, 오류가 많이 발생할 수 있기 때문에, 이를 간소화 하는 작업 이 필요하다. 본 연구에서는 가장 일반적인 간소화 방 법으로, 격자 지도를 생성하는 방법을 제안한다. 격자 지도를 생성하기 위한 확률은 수식(1)과 같다.

$$
p_{i}=\frac{N_{i}}{C_{i}}
$$

수식(1)에서 $i$ 는 격자지도의 한 격자를 의미하고, $N_{i}$ 는 그 격자 지도에 들어온 포인트의 수, $C_{i}$ 는 그 격자 지도에 들어올 수 있는 최대 포인트의 수를 의미 한다. $C_{i}$ 는 다음과 같다.

$$
C_{i}=\frac{\alpha}{s \times d_{i}}\left(\text { 단, } d_{i}<\mathrm{T}_{d}\right)
$$

$s$ 는 레이저 거리 센서의 각해상도를 의미하고, $d_{i}$ 는 차량의 위치와 격자 $(i)$ 사이의 거리를 의미한다. $\alpha$ 는 상수이다. 거리 $d_{i}$ 의 임계치 $\left(\mathrm{T}_{d}\right)$ 는 센서의 각해상 도 $(s)$ 에 의해 결정된다.

격자 지도를 생성하기 위한 확률이 높을수록 해당
격자는 객체가 있는 것으로 판단되고, 낮을수록 객체가 없는 것으로 판단하여 격자지도를 생성한다.

\section{2 보행자 모델}

일반적인 주행 차량의 경우, 센서는 차량의 앞 범 퍼에 해당되는 곳에 위치하게 된다. 센서가 낮은 부분 에 위치하여 있기 때문에, 이러한 센서로 보행자를 탐 색할 경우, 보행자의 무릎보다 조금 높은 위치를 탐색 하게 된다. 이러한 경우, 데이터가 좌측, 우측 발을 각 각 탐색하게 되고, 각 발을 서로 다른 이동 객체로 인 식하게 된다. 보행자의 좌측, 우측 발을 각각 탐색하게 되면, 동적 객체의 움직임이 일정하지 않게 된다. 이는 객체를 추적하는 과정에 있어 오류를 발생시키게 되고, 움직임을 파악하여 회피주행을 할 경우에도 오류를 발 생시킬 수 있다.

이러한 이유로 Jae Hoon Lee ${ }^{[8]}$ 은 보행자의 보행 방법에 따른 보행자 모델을 만들고, 보행자 모델을 바 탕으로 보행 모델을 제안하였다. 그러나 이 방법은 보 행자의 발이 서로 가려지는 현상(Occlusion)이 발생할 경우 적용하기 매우 어려울 뿐만 아니라, 레이저 센서 와 보행자의 다양한 위치 관계에 따른 많은 데이터의 변화를 모두 모델링하기 매우 어렵다. 또한 이 방법은 소수의 보행자의 패턴에 적용하기에는 적합하나, 보행 자 수에 비례하여 계산량이 많아지는 단점을 가진다. 센서가 가진 오차도 무시할 수 없다. 센서와 보행자 사이에 일정 거리 이상(약 $5 \mathrm{~m}$ )만 떨어져도, 보행자를 탐지하는 센서의 탐지 각도도 매우 줄어들고, 데이터 오차도 많이 발생하게 된다.

본 연구에서는 이러한 문제를 해결하기 위하여, 센 서를 통하여 획득한 데이터를 분석하여, 기계 학습방법 을 이용한 객체 분류 방법을 제안한다. 다양한 장소에 서 다양한 방법으로 이동한 보행자를 추출한 후, 이 데이터를 학습하고 분류하는 방법을 제안한다. 분류 및 학습을 하기 위한 데이터 특징 모델은 다음과 같다.

$$
x_{n}=\left\{\begin{array}{llll}
s_{n} & h_{n} & v_{n} & d_{n}
\end{array}\right\}
$$

$x_{n}$ 은 격자 지도 내에 존재하는 객체 중, $n$ 번째 객 체의 보행자 모델을 의미하고, 이는 정규화된 객체의 크기 $\left(s_{n}\right)$, 정규화된 모양 $\left(h_{n}\right)$, 속력 $\left(v_{n}\right)$, 방향 $\left(d_{n}\right)$ 의 정 보로 구성된다. 보행자 모델을 만들기 위하여 그림 


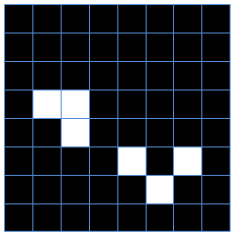

(a)

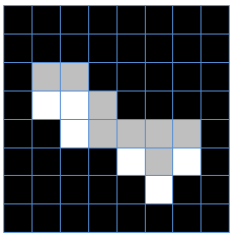

(b)

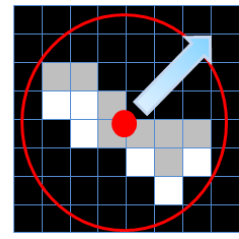

(c)
그림 1. 보행자 모델 및 그 생성 과정의 예. (a) 입력 데이터, (b) 모 폴로지 알고리즘 수행 결과, (c) 속도 및 방향 추출 결과

1(b)와 같이 격자 지도를 모폴로지(Morphology) 알고 리즘의 닫기(Closing) 방법을 사용하여 좌, 우측 발이 분리되지 않고 한 개의 객체로 보일 수 있게 처리한 후, 그림 1(c)와 같이 각 객체의 크기, 모양, 속도, 방 향 정보로 벡터를 구성한 후, 이를 분류기를 사용하여 분류한다.

\section{3. 객체 이동성 판단}

이동하는 차량에 장착된 레이저 센서를 통하여 데 이터를 획득할 경우, 획득한 데이터에서 객체의 이동성 을 파악하는 작업이 필요하다. 이를 파악하지 아니하 고 모든 물체들을 탐지 및 추적할 경우, 실시간으로 이를 파악하기 어려울 정도로 계산 복잡도가 증가하기 때문이다. 본 연구에서는 객체의 이동성을 측정하기 위 하여 물체의 이동 궤적을 이용하는 방법을 제안한다.

물체의 궤적을 이용할 경우, 차량의 위치정보를 같 이 활용하기 때문에, 움직이는 차량에서도 동적 객체와 정적 객체를 효율적으로 구분할 수 있는 장점을 가진 다. 일반적으로 동적 장애물 탐지 연구 방법들이 가지 는 문제점으로, 동적 장애물이 차량과 동일한 속도로 움직일 경우, 이를 동적 장애물로 탐지하기 어렵다는 문제를 가진다. 그러나 차량의 위치정보와 물체의 궤적 을 함께 사용하는 본 방법의 경우, 이러한 문제를 해 결할 수 있다.

이동 궤적은 그림 2 의 방법으로 이동 궤적을 구하 는 방법을 제시한다. 이 방법을 수식으로 표현하면 다 음과 같다.

$$
T_{i}^{n}=g \otimes G_{i-1}^{n}+g \otimes G_{i}^{n}+T_{i-1}^{n}
$$

$T_{i}^{n}$ 는 $i$ 번째 시간에 격자 지도에서 $n$ 의 위치에 있 는 물체의 이동 궤적을 의미하고, $g$ 는 스무딩 필터

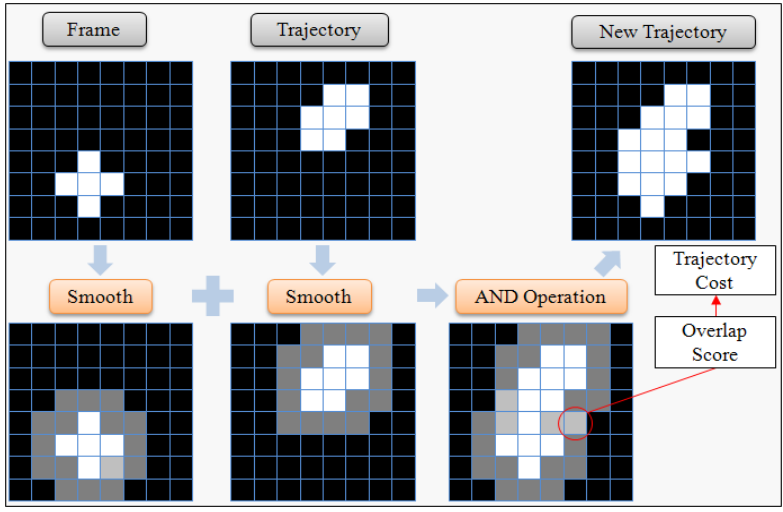

그림 2. 객체의 이동 궤적을 구하는 방법

(Smooth Filter)를 의미한다. $G_{i}^{n}$ 는 $i$ 번째 시간의 격자 지도를 의미한다. 스무딩 필터의 파라미터는 궤적 값 (Trajectory Cost)에 따라 부여되며, 이 궤적 값은 궤적 을 합칠 때, 영역이 겹쳐지는 정도를 의미한다. 영역이 합쳐지는 정도(Overlap Score)는 Taketoshi Mori ${ }^{[9]}$ 의 방법과 매우 유사하나, 그는 객체의 주위 격자만을 고 려하였고, 본 방법은 객체의 이동 속도에 따라 더 많 은 격자를 고려한다는 점에서 차이를 가진다. 영역이 합쳐지는 정도를 구하는 방법은 수식(5)와 같다.

$$
\operatorname{score}\left(G_{i}^{n}, T_{i-1}^{n}\right)=\sum_{k=0}^{K} \alpha_{k} n u m^{k}
$$

Taketoshi Mori의 연구 방법은 수식 (5)의 식에서 $K=2, \alpha_{0}=2, \alpha_{1}=1$ 로 계산하지만, 본 방법은 객체 가 움직이는 속도에 따라서 $K$ 값을 조절하는 방법을 사용하였다. 즉, 객체가 움직이는 속도가 빠를 경우, $K$ 값이 증가하고, 반대의 경우 감소한다.

수식 (5)에서 num 은 겹치는 격자의 수를 의미하고, $\alpha_{k}$ 는 상수로, 실험을 통하여 구한 값을 사용하였다.

수식 (4)를 이용하여 추출한 궤적을 이용하여 객체 의 이동성을 측정하기 위해, 객체의 모습에서 특징을 추출하여 이를 분류기를 사용하여 구분하는 방법을 활 용한다. 즉, 궤적과 그 안에 위치하는 현재 객체의 모 습을 바탕으로 물체가 이동하는 물체인지 정지하는 물 체인지를 판단한다.

객체와 궤적의 모습을 격자 지도에 형성한 후, 수 식 (6)의 방법으로 그 특징을 구성한다. 


$$
O_{i}^{n}=\left\{\begin{array}{lllllll}
A_{o} & A_{T} & R_{o} & R_{T} & \operatorname{Tr}_{o} & \operatorname{Tr}_{T} & P_{(o, T)}
\end{array}\right\}
$$

$i$ 번째 시간에 있는 $n$ 번째 객체의 모델 $\left(O_{i}^{n}\right)$ 은 객체 와 궤적의 넓이 $\left(A_{o}, A_{T}\right)$, 종횡비(Aspect Ratio, $\left.R_{o}, R_{T}\right)$, 둘레의 길이 $\left(T r_{o}, T r_{T}\right)$, 궤적에 대한 객체의 비율 $\left(P_{(o, T)}\right)$ 로 이루어진다. (o : 객체, $T$ : 궤적) 이 렇게 구한 객체의 모델은 이동성이 있는 객체, 이동성 이 없는 객체로 분류되며, 분류기를 통하여 이를 분류 한다.

\section{4. 객체 추적}

객체 $(O)$ 가 이동성이 있는 객체로 분류한 뒤, 분류 된 객체를 추적하는 방법은 아래 수식 (7)의 사후 확 률을 최대화시키는 문제로 생각할 수 있다.

$$
O^{*}=\underset{o}{\operatorname{argmax}} P(O \mid Z)
$$

베이시안 사후 확률은 다음과 같이 베이시안 공식 으로 해석할 수 있다.

$$
P(O \mid Z) \propto P(O) P(Z \mid O)
$$

즉, 사후 확률은 사전 확률(prior)과 우도(likelihood) 로 표현할 수 있다.

모든 객체들이 서로 독립적이라고 가정하면, 사전 확률은 각 객체들의 사전확률의 곱으로 정의할 수 있다.

$$
P(O)=\prod_{n=1}^{N} P\left(O^{n}\right)
$$

각 객체는 동일한 시간에 있는 다른 객체와 독립적 이고, 서로 다른 시간에 있는 객체와도 독립적이기 때 문에, 사전 확률은 각 객체가 가지고 있는 모양, 크기 정보를 모두 독립적으로 반영한다. 본 연구에서는 각 객체가 가지고 있는 모양과 크기 정보만을 이용하여 사전확률로 사용한다.

우도는 객체와 데이터 간의 물리적인 거리 차이를 사용하였다. 즉, 객체에 있는 데이터와 데이터와의 거 리가 가까울수록 우도 값은 커지고, 멀수록 우도 값은 작아진다. 그러나 단순히 객체에 있는 데이터와 입력으
로 들어오는 데이터와의 거리만 이용하였을 경우, 센서 에서 생기는 오차 값과, 객체에 포함되어 있는 다른 데이터의 값을 반영하지 않기 때문에, 보행자 객체 모 델의 중심점과 데이터와의 거리 사이의 값은 가우시안 함수의 형태로 우도 값을 정의한다.

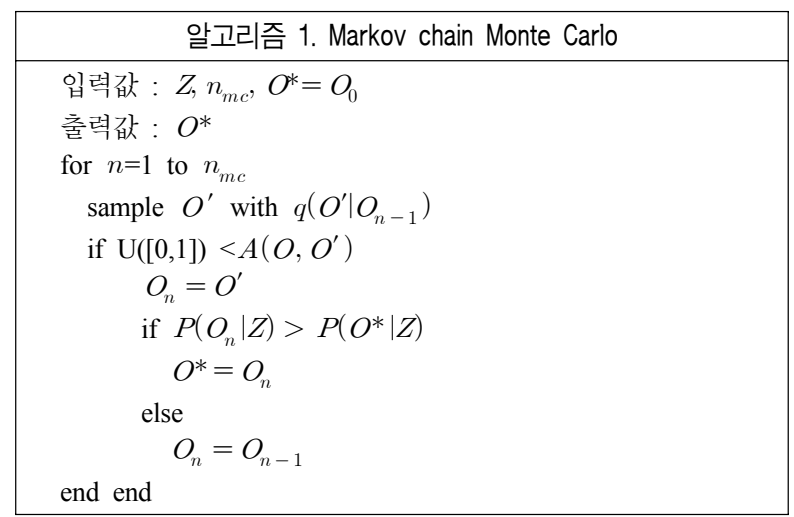

본 연구에서는 수식(8)을 계산하기 위한 방법으로 Markov chain Monte Carlo(MCMC)알고리즘을 제안한 다. 레이저 데이터는 연속적인 데이터이지만 주기적으 로 반복하는 데이터가 아니기 때문에, $\mathrm{MCMC}$ 알고리 즘을 사용할 수 있는 조건을 만족한다. $\mathrm{MCMC}$ 알고리 즘은 가장 적합한 분포 $(\pi(\omega))$ 를 추출할 수 있는 방법 으로, 본 연구에서 추출하고자 하는 분포는 $P(O \mid Z)$ 이다. 이를 계산하기 위한 가상 코드를 [알고리즘 1]에 서 제시한다.

\section{5. 실험 및 결과}

실외 환경에서 자율주행을 하기 위하여 전동 스쿠 터 시스템을 구축하고, 레이저 거리 센서와 IMU 센서 를 이용하여 데이터를 획득하였다.

실험을 위해 사용한 레이저 거리 센서는 $\mathrm{SICK}$ 사의 LMS151 모델로, 전방 $180^{\circ}$ 를 $25 \mathrm{~Hz}$ 의 속도로 스캔이 가능하다. 레이저 거리 센서의 위치는 그림 3 과 같이 설치하였다. 상단의 레이저 센서는 차량 진행 방향의 노면 정보를 수집하고 하단의 레이저 센서는 보행자 및 장애물 탐지에 필요한 데이터를 수집하여 스쿠터 주변의 환경을 인식할 수 있게 하였다.

격자 지도를 생성하기 위해서 사용한 격자의 사이 즈는 $10 \mathrm{~cm} \times 10 \mathrm{~cm}$ 을 사용하였고, 격자 내에 들어가는 최대 포인트의 수를 구하기 위한 파라미터 $(\alpha)$ 는 45 , 


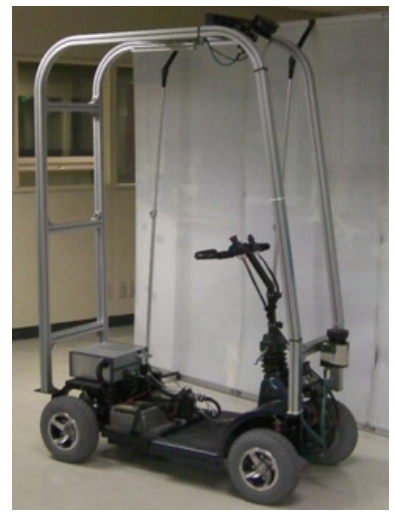

그림 3. 자율주행 차량

각 해상도는 $5^{\circ}$ 로 사용하였다.

보행자 모델을 학습하기 위하여 사용한 파라미터의 실험 방법은 다음과 같다. 객체 크기 $\left(s_{n}\right)$ 는 은 제안하 는 격자 지도에서 객체가 차지하는 격자의 수를 사용 하였고, 정규화된 모양 $\left(h_{n}\right)$ 은 객체를 포함하는 최소 포 함 사각형 변의 길이 비율을 사용하였다. 이와 같이 속도와 방향의 정보를 사용하여, 총 4 차원의 모델 데 이터를 사용하였다.

보행자 모델을 학습하기 위한 보행자는, 차량 주행 시 차량의 전방에서 다양한 방향, 다양한 속도로 이동 한 보행자의 데이터 약 500개를 사용하였으며, 각 모 델의 검증자료는(Ground-Truth) 사람이 직접 추출한 결 과를 사용하였고, 이를 학습 및 분류하기 위하여 SVM 분류기를 사용하였다.

보행자 탐지 성능은, 전체 4689개의 프레임 내에 존재하는 모든 객체(40328개) 중에서 보행자 객체의 수 (1639개) 보다 3배가량 많은 5208개가 검출되었다. 검출한 보행자 모델의 궤적을 추출하여 최종적으로 검 출한 움직이는 보행자를 총 1431 개 검출하여, $87.31 \%$ 의 탐지율을 보였다.

객체의 이동성을 추출하기 위하여 보행자 모델의 분류를 위하여 추출한 약 500 개의 보행자 모델에서 약 400 개의 이동 중인 보행자 정보를 사용하여 학습하였 다. 또한 궤적 추출을 위하여 사용한 프레임은 현재 프레임에서부터 이전 10 프레임을 사용하다. 보행자 모델을 학습 및 분류하기 위하여 SVM 분류기를 사용 하였다.

실험은 차량에 장착되어있는 $\mathrm{PC}(\mathrm{Dual}$ Core $2.3 \mathrm{Ghz}, 2 \mathrm{~GB} \mathrm{RAM})$ 에서 이루어졌으며, 수행 속도는
최소 $25 \mathrm{~Hz}$ 의 결과를 얻었다.

실험은 이동 객체 탐지율과 추적율을 도출하였으며, 이를 위해서 자체 제작한 데이터베이스에서 총4689개 의 프레임에서 검증 자료(Ground-Truth)를 추출하여 실 험을 진행하였다. 실험 결과, $87.31 \%$ 의 탐지율과 $75 \%$ 의 추적율의 결과를 보일 수 있었다. 실험을 위해 사 용한 탐지율은, 동적 객체를 올바로 탐지한 수를 전체 동적 객체 수로 나눈 값이고, 추적율은 실제 경로와 일 치하는 정도가 $80 \%$ 이상인 궤적의 비율을 사용하였다.

그림 4는 차량이 움직일 때, 올바르게 탐지 및 추 적한 추적 결과이다. 약 1 초 동안 차량이 움직이면서 획득한 데이터를 바탕으로 처리한 결과를 보여준다. 동 심원의 중앙이 현재 이동하고 있는 차량의 위치이다. 보행자 탐지 결과는 네모 박스로 표하였다.

그림 5 는 보행자를 잘못 인식한 결과이다. 차량의 이동방향 전면에 검출된 보행자로 인해, 후방에 있는 데이터에 가려짐 현상(Occlusion)이 발생하여, 잘못된 궤적을 형성하고, 이에 따른 오인식이 발생한 경우이 다. 본 알고리즘은 물체의 이동 궤적을 사용함으로, 차 량 주행 중에 움직이는 객체 탐지에 용이하나, 가려짐 현상에 약한 단점을 가진다.

실험에 사용한 검증 자료에서는 객체가 나타나는 순간부터 객체로 판단되지만, 본 연구 방법은 어느 정 도 이동한 후 그 궤적을 추출하여 물체의 이동성을 판 단하기 때문에, 일정 시간이 흐른 후에(약 0.3초)부터

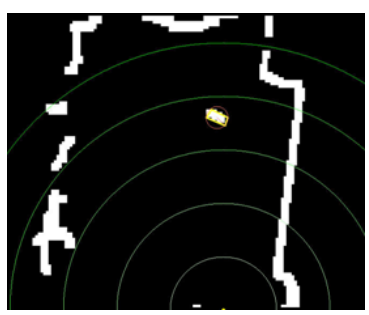

(a) 이동 객체 탐지

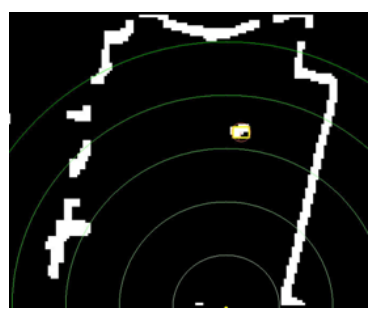

(c) 0.6 초 후

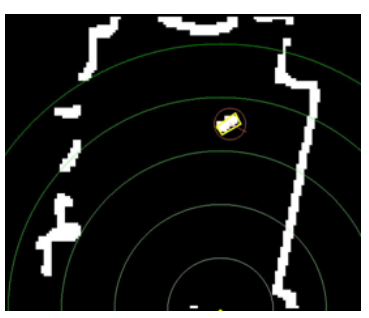

(b) 0.3 초 후

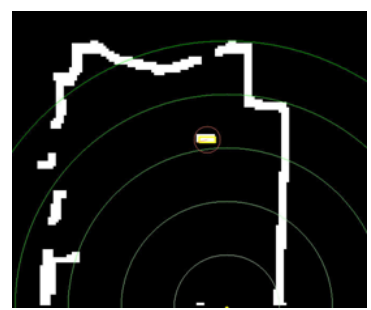

(d) 1초 후
그림 4. 알고리즘 수행 결과 (보행자 : 노란 네모 박스로 표시) 


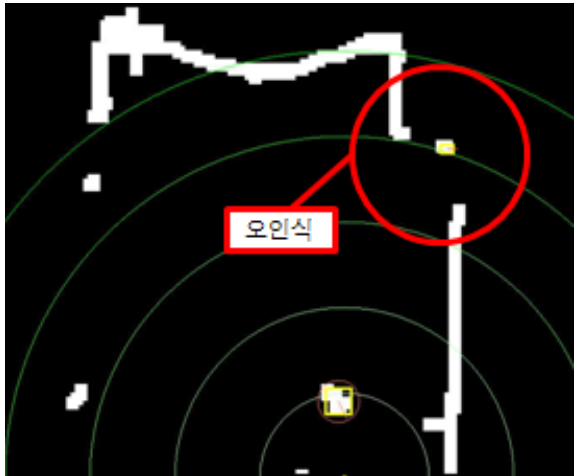

그림 5. 오인식

동적 객체를 파악하게 된다. 이로 인하여 탐지율이 다소 감소된다.

\section{6. 결 론}

본 논문에서는 자율주행 차량의 LRF 센서 데이터 를 이용하여 주행 방향에 존재하는 동적 객체를 탐지 및 추적하는 방법을 제안하였다. 이를 위하여 보행자 모델을 제시하였으며, 이 보행자 모델을 $\mathrm{MCMC}$ 알고 리즘을 활용하여 추적하는 방법을 제안하였다. 또한, 실제 자율주행 차량에 탑재한 실험을 통하여 $87.31 \%$ 의 탐지율과 $75 \%$ 의 추적율 결과를 도출하였다.

본 연구 방법은 물체의 이동 궤적을 이용하였기에, 주행 차량의 절대적, 상대적 위치가 매우 정확하게 추 출되어야 그 이동 궤적을 정확하게 추출할 수 있다는 단점을 가진다. 최근에는 레이저 센서 데이터를 이용하 여 이러한 위치 정보를 추출하는 것과(SLAM) 장애물 을 찾는 것을 동시에 진행하는 연구가 많이 이루어지 고 있다. 본 연구 방법도, 이러한 연구 방향을 반영하 여, 레이저 센서 데이터를 이용한 위치 정보 추출 방 법을 병합할 경우, 더 정확한 처리 결과를 볼 수 있을 것으로 예상된다.

\section{참고문헌}

[1] Trung-Dung $\mathrm{Vu}$ and Olivier Aycard, "Lased-based detection and tracking moving object using data-driven markov chain monte carlo", In IEEE International Conference on Robotics and Automation (ICRA), pp3800-3806, May, 2009.
[2] C.C. Wang, "Simultaneous Localization, Mapping and Moving Object Tracking". $\mathrm{PhD}$ thesis, Robotics Institute, Carnegie Mellon University, Pittsburgh, PA, April 2004.

[3] A. Petrovskaya and S. Thrun, "Model based vehicle tracking for autonomous driving in urban environments", In Proceedings of Robotics: Science and Systems IV, June 2008.

[4] M. Darms, P. Rybski, and C. Urmson, "Classification and tracking of dynamic objects with multiple sensors for autonomous driving in urban environments", Intelligent Vehicles Symposium, pp.1192-1202, June 2008.

[5] Lee Jae-Yeong and Yu Wonpil, "Moving object tracking in driving environment", Ubiquitous Robots and Ambient Intelligence (URAI), pp. 139-141, Nov 2011.

[6] S. Bota and S. Nedesvchi, "Multi-feature walking pedestrians detection for driving assistance systems.", Intelligent Transport Systems, Vol.2, No.2, pp.92-104, 2008.

[7] Chung-Kyeom Kim, Young-Min Han, Byung-Hyu Bae and Jung-Ha Kim, "The research of path planning algorithm considering vehicle's turning radius for unmanned ground vehicle", International Conference on Control, Automation and Systems (ICCAS), pp. 754-756, 2011.

[8] Jae Hoon Lee, Takashi Tsubouchi Kenjiro Yamamoto and Saku Egawa, "People Tracking Using a Robot in Motion with Laser Range Finder", International Conference on Intelligent Robots and Systems, pp. 2936-2942, 2006.

[9] T. Mori, Takahiro Sato, H. Noguchi, M. Shimosaka, R. Fukui and Tomomasa Sato, "Moving Objects Detection and Classification Based on Trajectories of LRF Scan Data on a Grid Map”, International Conference on Intelligent Robots and Systems, pp.2606-2611, 2010 . 


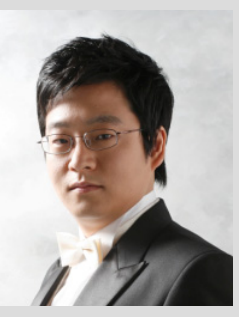

2006 경인교육대학교교육학 과 (교육학 학사)

2008 숭실대학교미디어학과 (공학석사)

2010 한양대학교 전자컴퓨터 통신공학과 박사과정 (수료)

2011 현재 한국과학기술연구원 연구원 관심분야: Computer vision, Image processing

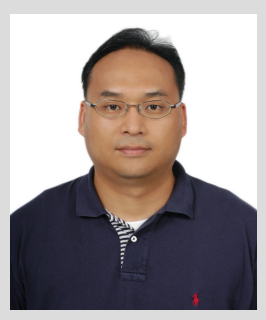

\section{김 창 환}

1993 한양대학교 기계공학과 (공학사)

1995 한양대학교 기계설계공 학과 (공학 석사)

2002 미국 아이오와주립대기 계공학과 (공학박사)

2004 미국 노틀담대학교 항공 기계공학과 박사후 연구원 (Research Association) 2004 현재 한국과학기술연구원 책임연구원 관심분야: Robotics

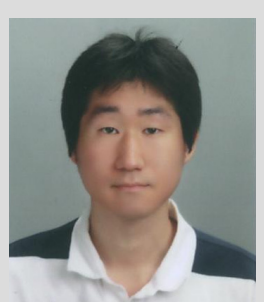

김 남 훈

2006 고려대학교 전기전자전 파공학부(공학사)

2009 KAIST 로봇공학학제 전공(공학석사)

2010 현재 한국과학기술연 구원 연구원

관심분야 : Robotics, Artificial Intelligence

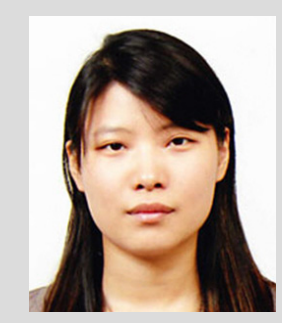

\section{윤 정 연}

2009 경희대학교 컴퓨터 공학 화(공학사)

2010 현재 한국과학기술연구 원 연구원

관심분야 : Mobile Robot, $\mathrm{HCI}$ 